\title{
A Novel Probabilistic Fatigue Life Prediction Method for Welded Structures Based on gPC
}

\author{
Huiying Gao, Xiaoqiang Zhang $\mathbb{D}$, Xiaoqiang Yang, and Bo Zheng \\ Aviation Engineering Institute, Civil Aviation Flight University of China, Guanghan 618307, China \\ Correspondence should be addressed to Xiaoqiang Zhang; xqzhanguestc@163.com
}

Received 20 February 2021; Revised 4 April 2021; Accepted 20 April 2021; Published 26 April 2021

Academic Editor: Samuel Yousefi

Copyright (C) 2021 Huiying Gao et al. This is an open access article distributed under the Creative Commons Attribution License, which permits unrestricted use, distribution, and reproduction in any medium, provided the original work is properly cited.

\begin{abstract}
The traditional fatigue life prediction methods based on the $\mathrm{S}-\mathrm{N}$ curve all believe that the parameters in the model are deterministic constants and can be categorized to the deterministic life prediction. However, in practice, it is difficult to carry out a large number of experiments due to the limitation of time or the possible shortage of funds. In addition, the specimens used in the experiments are not exactly the same, and the test operations and data reading depend on the accuracy of the test equipment as well as the subjective judgment of the testers, which result to the uncertainty of the S-N curve. Therefore, the uncertainty should be considered in order to improve the accuracy of the fatigue life prediction. In this paper, the uncertain factors affecting the fatigue life of welded joints are summarized, and the generalized polynomial chaos (gPC) is introduced into fatigue life prediction. A novel probabilistic fatigue life prediction method combined with the nonlinear cumulative damage model considering the uncertainty of the S-N curve is constructed. An illustrative example is presented to demonstrate the advantages of the proposed approach.
\end{abstract}

\section{Introduction}

The S-N curve is mainly obtained through a large number of fatigue tests and fitting analysis. Many fatigue life prediction methods based on the S-N curve believe that the parameters in the life prediction models are deterministic, that is, in a given loading environment, each parameter is a constant. Since this kind of methods considers the parameters in the models as constants, it is known as deterministic life prediction methods. In practice, these assumptions are not always true. Due to the influence of uncertain factors such as test samples, conditions, operations, and data reading, the fatigue life under constant amplitude load is often dispersed in different degrees, which leads to the uncertainty of the $\mathrm{S}-\mathrm{N}$ curve. For welded joints, the S-N curve is affected not only by external uncertainties such as load and environment but also by internal factors such as the welding process, joint form, residual stress, and welding defects. The internal factors, such as welding defects, are the important causes of stress concentration. Under a cyclic loading, stress concentration usually becomes the birthplace of fatigue cracks, and it will affect the fatigue life of the welded structure.
Generally, the shape, size, and stress distribution of the welded structure are uncertain, resulting in the uncertainty and dispersion of fatigue life. Even for the same type of welded joints and the same welding materials, different S-N curves will be obtained while different welding methods are used. $\mathrm{Xu}$ and Zhang [1] studied the fatigue properties of a TC4 thin plate of T-welded joint and found that the weld toe or weld surface was the most frequent location of fracture initiation and different welding processes had different characteristics. As a result, the welded joints with the same welding materials and joints have different S-N curves. Jonsson et al. [2] studied the fatigue performance of the welded bogie joint under different welding process parameters, that is, by changing the current and voltage, it was found that the static load strength and the shape of the joint were not sensitive to the change of welding parameters, but the fatigue limit decreased with the increase of current. There are many kinds of welded joints used in complex mechanical structures including butt joint, lap joint, and cross joint. The notch forms for each type of welded joints are different, and the degree of nonuniformity of stress distribution is usually different. For example, the stress 
distribution of the butt joint is relatively uniform while it is nonuniform for the cross joint because of the sharp transition from the weld to the base metal. For lap joints, the nonuniformity of stress distribution is reflected not only in the weld but also in the lap plate [3]. The nonuniform stress distribution will lead to stress concentration, and the stress concentration factors are quite different for each form of welded joints.

Up to now, scholars have proposed many probabilistic fatigue life prediction models for various uncertainties. The main theoretical bases of these models include probability and statistics, entropy theory, Bayes theory, fuzzy theory, and rough set theory.

The method of fatigue life prediction based on probability and statistics randomizes the relevant parameters in the models, including load, material parameters $[4,5]$, and structural size [6], and describes the objective uncertainty as a variable that obeys a random distribution. There are two ways to deal with this kind of methods. One is to randomize the fatigue life or damage under constant amplitude load. Rao et al. [7] considered the uncertainty of stress range and material properties and adopted the probabilistic S-N curve and a fatigue cumulative damage model to obtain the distribution of fatigue life. Shen et al. [8] considered the uncertainty of the loading process and the fatigue strength of the material, randomized the damage caused by a single cycle, and found the relationship between the distribution function and the stress amplitude. Liu and Mahadevan [9] regarded the fatigue life under different constant amplitude loads as random processes, and Karhunen-Loeve expansion was used to describe the variability of the corresponding random processes. $\mathrm{Ni}$ and Mahadevan [10] combined the probabilistic Miner rule and random stress-strain curve family to propose an energy-based probabilistic fatigue life prediction method under multiaxial variable amplitude loading. The other way is to randomize the parameters such as load, material properties and structural dimensions in the life prediction model. Based on the Kitagawa-Takahashi diagram, Liu and Mahadevan [11] proposed that the equivalent initial flaw size (EIFS) was only related to the fatigue limit and the threshold value of stress intensity factor. Maljaars et al. [12] predicted probabilistic fatigue crack growth of welded joints in civil engineering structures by randomizing the parameters in the model and considering the relative importance of random variables. Luo et al. [13] considered the effects of crack nucleation life and crack growth resistance variability and proposed a framework for fatigue life prediction based on crack nucleation and surface defects.

Entropy theory is widely used in medicine, environment, management, statistics, physics, and so on. The main applications for entropy in probabilistic fatigue life prediction are maximum entropy and thermodynamic entropy. Yi et al. [14] proposed a method to determine the type of probability distribution of fatigue life and describe the dispersion of fatigue life. Guan et al. [15] proposed the overall framework of probabilistic fatigue damage prediction based on the maximum correlation entropy method and found that this method can give satisfactory prediction results. In addition, the maximum correlation entropy theory and Bayes theory are combined to provide support for health management decision making [16]. Thermodynamic entropy theory is also used to predict fatigue life. Zhu et al. [17] proposed a probabilistic fatigue life prediction model combining with thermodynamic entropy theory and Bayes theory. Naderi and Khonsari [18] used the theory of thermodynamic entropy to realize the real-time monitoring of fatigue life. Temfack and Basaran [19], Wang and Yao [20], and Kim et al. [21] also applied entropy theory to life prediction.

The probabilistic fatigue life prediction method based on Bayes theory is another common method to deal with uncertainties. Guan et al. [22] adopted Bayes update to reduce the uncertainty in fatigue damage prediction and then used MCMC (Markov Chain Monte Carlo) simulation to select and update models and predict fatigue damage with mean probability. An et al. [23] introduced field failure data into prior information and adopted Bayes theory combined with MCMC simulation to obtain the parameters of fatigue life distribution. Chookah et al. [24] proposed a probabilistic failure physical model considering pitting fatigue degradation mechanism. The probability density function was used to represent the uncertainty of the parameters of the proposed model, and the Bayes theory combined with experimental data was used to evaluate the model. Zárate et al. [25] proposed a theoretical framework for updating and predicting crack length based on Bayes theory. The theoretical framework includes two main contents: the model update part, which is used to identify the probability density function of fracture mechanics parameters, and the prediction part, which is used to predict the crack length of the specimen and regards it as a function related to the number of cycles. Huang et al. [26], Mustafa et al. [27], Zhu et al. [28], and so on have been working on fatigue life prediction based on Bayes theory and have achieved many results.

The probabilistic fatigue life prediction method based on fuzzy theory introduces fuzzy sets and membership functions to deal with the uncertain phenomena in fatigue life prediction. Since it was put forward, many models and methods have emerged. Muc [29] established the uncertainty model of fatigue life by using the fuzzy set method combined with preselection of material parameters. Nopiah et al. [30] identified low fatigue damage by using typical statistical characteristics based on fuzzy theory. Bhalla et al. [31] established a fuzzy probabilistic damage model to predict the residual life of bolted joints. Zhu et al. [32] proposed a fatigue life prediction model by the fuzzy set method.

In addition to the abovementioned theories, there are other theories and methods that can be applied to probabilistic fatigue life prediction, such as rough set theory $[33,34]$ and grey theory [35].

Among the abovementioned methods, the fatigue life prediction method based on probability and statistics is obtained on the basis of a large number of experiments and has high credibility. The life prediction method based on entropy theory, rough set theory, and grey theory has a short history and needs to be further discussed. Compared with the abovementioned methods, the development course of 
fatigue life prediction based on Bayes theory and fuzzy mathematics is longer while the maturity of them is not enough, and further research is needed.

Although a lot of research works have been carried out, some theories and methods which have strong ability to deal with uncertain problems, such as polynomial chaos theory [36], evidence theory [37], and concept lattice theory [38], are rarely reported in the field of fatigue life prediction. In this paper, a novel probabilistic fatigue life prediction method combined with the nonlinear cumulative damage model based on $\mathrm{gPC}$ is proposed.

The remainder of this paper is organized as follows. Section 2 introduces the generalized polynomial chaos. Section 3 discusses the uncertainties and proposes a novel probabilistic fatigue life prediction method. Section 4 presents an illustrative example. Conclusions are finally summarized in Section 5.

\section{The Generalized Polynomial Chaos (gPC)}

The main idea of polynomial chaos theory is to approximately represent a random process by the sum of orthogonal polynomials corresponding to a specific distribution type. It was first proposed by Wiener [39] to establish a turbulence model. The polynomial chaos method belongs to the nonstatistical method. Compared with the traditional statistical method, the polynomial chaos method has the advantages of small amount of calculation and high accuracy. Because of these advantages, scholars applied this method to various engineering fields, such as finite deformation problems, transient heat transfer problems, and stochastic difference equations. At present, the polynomial chaos theory mainly includes generalized polynomial chaos (gPC), multielement generalized polynomial chaos (ME-gPC), and arbitrary polynomial chaos (aPC). Compared with gPC, ME-gPC can deal with the discontinuity of random space, and aPC can carry out polynomial chaos expansion while the uncertain parameters are distributed arbitrarily, but gPC has a longer development time and is more widely used and more mature.

We suppose a model

$$
Y=\kappa(z) .
$$

Assuming that $Y$ cannot be expressed analytically by $z$, but the probability space of $z$ is smooth sufficiently, and the response $Y$ corresponding to $z$ can be obtained by numerical simulation, the relevant statistical properties of $Y$ can be obtained by the surrogate model. For a system, if the input is affected by random parameters represented by the set $\left\{\xi_{i}\right\}_{i=1}^{n}$, then, since the uncertainty of the output is only determined by the input, the output of the system can also be represented by the set $\left\{\xi_{i}\right\}_{i=1}^{n}$. For the random field of Gaussian distribution, the series of Wiener-Askey polynomial chaos converges exponentially, so Wiener polynomial chaos can well deal with the problem of Gaussian distribution random field. For the random field with non-Gaussian distribution, Ghanem [40] proved that the series of Wiener polynomials chaos also converges, but the speed is relatively slow. In order to solve this problem, Xiu [41] and Sepahvand [42] extended Hermite polynomials and finally obtained a family of polynomial chaos for random variables with different distribution types. The polynomial chaos families corresponding to common distribution types are shown in Table 1.

Assuming that the input random variables of the system follow a Gaussian distribution, the polynomial chaos corresponding to the Gaussian distribution can be expanded as

$$
\begin{aligned}
Y= & \alpha_{0} \Gamma_{0}+\sum_{i_{1}}^{\infty} \alpha_{i_{1}} \Gamma_{1}\left(\xi_{i_{1}}\right) \\
& +\sum_{i_{1}=1}^{\infty} \sum_{i_{2}=1}^{i_{1}} \alpha_{i_{1} i_{2}} \Gamma_{2}\left(\xi_{i_{1}}, \xi_{i_{2}}\right) \\
& +\sum_{i_{1}=1}^{\infty} \sum_{i_{2}=1}^{i_{1}} \sum_{i_{3}=1}^{i_{2}} \alpha_{i_{1} i_{2} i_{3}} \Gamma_{3}\left(\xi_{i_{1}}, \xi_{i_{2}}, \xi_{i_{3}}\right)+\cdots,
\end{aligned}
$$

where $\alpha_{i}(i=1,2,3, \ldots)$ are the undetermined constant coefficients, $\xi_{j}(j=1,2,3, \ldots)$ are independent variables and obey the standard normal distribution, and $\Gamma_{k}\left(\xi_{i_{1}}, \xi_{i_{2}}, \ldots, \xi_{i_{k}}\right)$ is the $k$ th multidimensional Hermite polynomial.

For simplicity, equation (2) can be written in a compact form:

$$
Y=\sum_{j=0}^{\infty} \beta_{j} \Pi_{j}(\xi)
$$

where $\beta_{j}$ is the coefficient of polynomial, $\Pi_{j}(\xi)$ is the Wiener-Askey polynomial chaos, and $\xi=\left(\xi_{1}, \xi_{2}, \ldots, \xi_{n}\right)$ is the random variable vector.

Assuming that $\xi$ is a two-dimensional random variable vector, equation (2) can be rewritten as

$$
\begin{aligned}
Y= & \alpha_{0} \Gamma_{0}+\alpha_{1} \Gamma_{1}\left(\xi_{1}\right)+\alpha_{2} \Gamma_{1}\left(\xi_{2}\right) \\
& +\alpha_{11} \Gamma_{2}\left(\xi_{1}, \xi_{1}\right)+\alpha_{12} \Gamma_{2}\left(\xi_{1}, \xi_{2}\right) \\
& +\alpha_{22} \Gamma_{2}\left(\xi_{2}, \xi_{2}\right)+\alpha_{111} \Gamma_{3}\left(\xi_{1}, \xi_{1}, \xi_{1}\right)+\alpha_{112} \Gamma_{3}\left(\xi_{1}, \xi_{1}, \xi_{2}\right) \\
& +\alpha_{122} \Gamma_{3}\left(\xi_{1}, \xi_{2}, \xi_{2}\right)+\alpha_{222} \Gamma_{3}\left(\xi_{2}, \xi_{2}, \xi_{2}\right)+\cdots .
\end{aligned}
$$

Comparing equations (4) with (3), it can be seen that the function is changed only by the subscript. Therefore, equation (4) is equivalent to

$$
\begin{aligned}
Y= & \beta_{0} \Pi_{0}+\beta_{1} \Pi_{1}+\beta_{2} \Pi_{2}+\beta_{3} \Pi_{3}+\beta_{4} \Pi_{4}+\beta_{5} \Pi_{5}+\beta_{6} \Pi_{6} \\
& +\beta_{7} \Pi_{7}+\beta_{8} \Pi_{8}+\beta_{9} \Pi_{9}+\cdots .
\end{aligned}
$$

The expression of the $n$-order Hermite polynomial is

$$
\Gamma_{n}\left(\xi_{i_{1}}, \xi_{i_{2}}, \xi_{i_{3}}, \ldots, \xi_{i_{n}}\right)=e^{(1 / 2) \xi^{T} \xi}(-1)^{n} \frac{\partial^{n}}{\partial \xi_{i_{1}} \partial \xi_{i_{2}}, \ldots, \partial \xi_{i_{n}}} e^{-(1 / 2) \xi^{T} \xi}
$$

According to equation (6), it is easy to obtain a onedimensional Hermite polynomial. 
TABLE 1: The polynomial chaos corresponding to random variables of different distribution types.

\begin{tabular}{lc}
\hline Distribution type & Polynomial chaos type \\
\hline Gaussian & Hermite chaos \\
Uniform & Legendre chaos \\
Gamma & Laguerre chaos \\
Beta & Jacobi chaos \\
Poisson & Charlier chaos \\
Binomial & Krawtchouk chaos \\
Hypergeometric & Hahn chaos \\
Nonnegative binomial & Meixner chaos \\
\hline
\end{tabular}

$$
\begin{aligned}
& \Gamma_{0}(\xi)=1, \\
& \Gamma_{1}(\xi)=\xi \\
& \Gamma_{2}(\xi)=\xi^{2}-1, \\
& \Gamma_{3}(\xi)=\xi^{3}-3 \xi, \ldots, \Gamma_{n}(\xi)=\xi \Gamma_{n-1}(\xi)-(n-1) \Gamma_{n-2}(\xi) .
\end{aligned}
$$

Tables 2 and 3 show the one-dimensional and two-dimensional Hermite polynomial of each order, respectively.

If the random variables in Hermite polynomials follow the standard normal distribution and are independent from each other, then the polynomials are orthogonal, that is,

$$
\left\langle\Pi_{m}, \Pi_{n}\right\rangle=0, \quad m \neq n .
$$

Then, the Hermite polynomials form a set of complete orthogonal bases in 2-dimensional space, and it has

$$
\begin{aligned}
\left\langle\Pi_{m}, \Pi_{n}\right\rangle & =\left\langle\Pi_{m}^{2}\right\rangle \delta_{m n}, \\
\langle p(\xi), q(\xi)\rangle & =\int p(\xi) q(\xi) \theta(\xi) \mathrm{d} \xi,
\end{aligned}
$$

where $\langle\cdot, \cdot\rangle$ is the inner product, $\delta_{m n}$ is the Kronecker function, $\theta(\xi)$ is the weight function of the Hermite polynomial, and it satisfies

$$
\theta(\xi)=\frac{1}{\sqrt{2 \pi}} e^{-(1 / 2) \xi^{T} \xi} .
$$

The coefficient of Hermite polynomial $\beta_{j}$ in equation (3) can be obtained by Galerkin projection method, that is,

$$
\beta_{j}=\frac{\left\langle Y, \Pi_{i}\right\rangle}{\left\langle\Pi_{i}^{2}\right\rangle}=\frac{1}{\left\langle\Pi_{i}^{2}\right\rangle} \int Y \Pi_{i} \theta(\xi) \mathrm{d} \xi .
$$

This Galerkin projection method has some limitations; it requires that $Y$ must have a clear analytical solution. Therefore, the random response surface method will be adopted in this paper.

\section{The Proposed Probabilistic Fatigue Life Prediction Method}

3.1. The Uncertainty Analysis and Probabilistic Fatigue Life Prediction of the S-N Curve. For the life prediction method based on the S-N curve and fatigue cumulative damage theory, the S-N curve is crucial, which affects the prediction accuracy. In the case of a given stress ratio, the $\mathrm{S}-\mathrm{N}$ curve is
TABle 2: The Hermite polynomial of each order for a one-dimensional random variable.

\begin{tabular}{ccc}
\hline$j$ & The order $\chi$ & The $j$ th Hermite polynomial $\Pi_{j}$ \\
\hline 0 & $\chi=0$ & 1 \\
1 & $\chi=1$ & $\xi_{1}$ \\
2 & $\chi=2$ & $\xi_{1}^{2}-1$ \\
3 & $\chi=3$ & $\xi_{1}^{3}-3 \xi_{1}$ \\
4 & $\chi=4$ & $\xi_{1}^{4}-6 \xi_{1}^{2}+3$ \\
\hline
\end{tabular}

TABLe 3: The Hermite polynomial of each order for two-dimensional random variables.

\begin{tabular}{lcc}
\hline$j$ & The order $\chi$ & The $j$ th Hermite polynomial $\Pi_{j}$ \\
\hline 0 & $\chi=0$ & 1 \\
\hline 1 & $\chi=1$ & $\xi_{1}$ \\
2 & $\xi_{2}$ \\
\hline 3 & $\chi=2$ & $\xi_{1}^{2}-1$ \\
4 & $\xi_{1} \xi_{2}$ \\
5 & & $\xi_{2}^{2}-1$ \\
\hline 6 & & $\xi_{1}^{3}-3 \xi_{1}$ \\
7 & $\xi_{1}^{2} \xi_{2}-\xi_{2}$ \\
8 & & $\xi_{1} \xi_{2}^{2}-\xi_{1}$ \\
9 & & $\xi_{2}^{3}-3 \xi_{2}$ \\
\hline 10 & & $\xi_{1}^{4}-6 \xi_{1}^{2}+3$ \\
11 & $\xi_{1}^{3} \xi_{2}-3 \xi_{1} \xi_{2}$ \\
12 & & $\xi_{1}^{2} \xi_{2}^{2}-\xi_{1}^{2}-\xi_{2}^{2}+1$ \\
13 & & $\xi_{1} \xi_{2}^{3}-3 \xi_{1} \xi_{2}$ \\
14 & $\xi_{2}^{4}-6 \xi_{2}^{2}+3$ \\
\hline
\end{tabular}

tested under different levels of constant amplitude load, and the corresponding fatigue life of all levels of load is recorded and then fitted in the later stage. In the deterministic fatigue life prediction method, the " $\mathrm{N}$ " in the S-N curve usually refers to the average fatigue life under all levels of stress, without considering its dispersion. However, for welded structures and parts in engineering, this treatment method often cannot achieve satisfactory results and may even lead to catastrophic accidents.

The expression of the S-N curve is more commonly used in the form of power function, that is, the relationship between fatigue life and stress level is expressed by

$$
N_{f}(\Delta \sigma)^{m}=C
$$

where $N_{f}$ is the fatigue life cycle, $\Delta \sigma$ is the stress, and $C$ and $m$ are the material parameters.

Taking logarithms on both sides of the abovementioned equation, it can be transformed into

$$
\ln N_{f}+m \ln (\Delta \sigma)=\ln C .
$$

From equation (12), it is found that the fatigue life cycle $N_{f}$ will be affected by material parameters $C$ and $m$ under the action of steel. The study shows that, for a given material, the parameters $C$ and $m$ are not fixed constants, and there is a certain degree of dispersion and uncertainty, which is due to the uncertainty of inclusions, pores, and microcracks in the specimen. For welded joints, the internal welding defects are inevitable, including more pores, inclusions, and 
microcracks, than nonwelded structures, and the distribution is more complex than that of nonwelded structures. Therefore, when the method based on the S-N curve and fatigue cumulative damage theory is used to predict the life of welded joints, it is necessary to consider the uncertainty of the S-N curve which originates from the uncertainty of material parameters $C$ and $m$. In this paper, the gPC is proposed to consider the uncertainty of the $\mathrm{S}-\mathrm{N}$ curve and combine with the improved Manson-Halford model and the improved Corten-Dolan model to predict the probabilistic fatigue life.

The fatigue life under constant amplitude load is obtained on the basis of the S-N curve. Generally speaking, it is considered that the fatigue life $N(\mathbf{x})$ follows a lognormal distribution or a Weibull distribution. $\mathbf{x}=x_{1}, x_{2}, \ldots, x_{n}$ is the uncertain parameter vector of the fatigue life. For example, $N(\mathbf{x})$ follows a lognormal distribution, while $\ln (N(\mathbf{x}))$ follows a normal distribution. In order to establish the polynomial chaos expansion model of fatigue life, it is assumed that the uncertain parameters $x_{1}, x_{2}, \ldots, x_{n}$ are independent of each other. Therefore, the fatigue life corresponding to all levels of stress can be approximately calculated by

$$
\ln (N(\mathbf{x}))=\sum_{j=0}^{\infty} \beta_{j} \prod_{j}(\mathbf{x}), \quad \mathbf{x}=\left\{x_{1}, x_{2}, \ldots, x_{n}\right\} .
$$

According to the properties of the polynomial chaos expansion, the finite term can be used to approximately estimate the fatigue life. Assuming that the number of terms is $s$, the fatigue life prediction model can be simplified as

$$
\ln (N(\mathbf{x}))=\sum_{j=0}^{s-1} \beta_{j} \prod_{j}(\mathbf{x}), \quad \mathbf{x}=\left\{x_{1}, x_{2}, \ldots, x_{n}\right\},
$$

where $s=((n+\chi) ! /(n ! \chi !))$ and $\chi$ is the order of expansion for polynomial chaos.

In order to improve the speed of calculation, $x_{1}, x_{2}, \ldots, x_{n}$ can be normalized into random variables $\zeta_{1}, \zeta_{2}, \ldots, \zeta_{n}$

$$
\zeta_{i}=\frac{x_{i}-E\left(x_{i}\right)}{\sqrt{\operatorname{Var}\left(x_{i}\right)}}
$$

where $E\left(x_{i}\right)$ and $\operatorname{Var}\left(x_{i}\right)$ denote the expectation and variance of the random variable $x_{i}$, respectively. Since the random variables are independent from each other, after standardization, the random variables in $\zeta=\left(\zeta_{1}, \zeta_{2}, \ldots, \zeta_{n}\right)$ are independent from each other. The multidimensional orthogonal polynomial $\prod_{j}(\zeta)$ can be derived as

$$
\prod_{j}(\zeta)=\prod_{i=1}^{n} \varphi_{i}^{l_{i}}\left(\zeta_{i}\right)
$$

where $j=0,1,2, \ldots, s-1, \quad l_{i}=0,1,2, \ldots, \chi, \quad \sum_{i=1}^{n} l_{i} \leq \chi$. $\varphi_{i}^{l_{i}}\left(\zeta_{i}\right)$ is the $l_{i}$-order orthogonal polynomial, whose parameters are random variables $\zeta_{i}(i=1,2, \cdots, n)$. According to the distribution type of $\zeta_{i}$, the corresponding relations in
Table 1, the base of one-dimensional orthogonal polynomial $\varphi_{i}^{l_{i}}\left(\zeta_{i}\right)$ can be determined.

It is assumed that the fatigue life of the material under constant amplitude load $\Delta \sigma$ is expressed by $N(\mathbf{x})$. It is known that the material parameters $C$ and $m$ are random variables and $\mathbf{x}=(\ln (C), m)$. Then, the polynomial chaos of fatigue life under constant amplitude load can be obtained by substituting $\mathbf{x}=(\ln (C), m)$ into the abovementioned model and solving the coefficients of the polynomial chaos. The probabilistic fatigue life prediction model based on gPC is finally obtained. The whole calculation process is shown in Figure 1.

From Figure 1, it is known that the key point for the uncertainty analysis of the S-N curve and probabilistic fatigue life prediction based on $\mathrm{gPC}$ is solving the coefficients of the polynomial chaos. In this paper, the stochastic response surface method is used.

3.2. gPC-Based Stochastic Response Surface Method. As mentioned above, how to calculate the coefficients of the polynomial chaos $\beta_{j}$ is the fundamental and critical procedure. In the space expanded by the base vector of $\left\{\xi_{i}\right\}_{i=1}^{n}$, each set of determined sample value corresponds to a point named sampling point. For the model with multidimensional random variables, the common prescription is to accomplish polynomial chaos expansion and calculate the coefficients via choosing appropriate sampling points; then, the polynomial chaos of the model is obtained. The key of the stochastic response surface method is how to choose the appropriate sampling points. Taking the random field of Gaussian distribution as an example, it can be expanded into an approximate expression based on Hermite polynomials according to the Wiener polynomial chaos theory. It is assumed that the highest order in the expansion is $\chi$, and then, the roots of the $\chi+1$ order Hermite primary function can be taken as the collocation points.

Let $n=2, \chi=2$, that is, the model containing two-dimensional random variables is expanded to a second-order Hermite polynomial, then $s=(((\chi+n) !) /(\chi ! n !))=((2+2) ! /(2 ! 2 !))=6$, and the combinatorial number of the corresponding roots is $(\chi+1)^{n}=(2+1)^{2}=9$. The partial Hermite primary functions corresponding to the Gaussian distribution random field are shown in Table 4.

From Table 4, the root of the third-order Hermite primary function can be obtained, that is, $0, \sqrt{3},-\sqrt{3}$; thus, the corresponding sampling points include $(0,0),(0, \sqrt{3})$, $(0,-\sqrt{3}), \quad(\sqrt{3}, 0), \quad(\sqrt{3}, \sqrt{3}), \quad(\sqrt{3},-\sqrt{3}), \quad(-\sqrt{3}, 0)$, $(-\sqrt{3}, \sqrt{3})$, and $(-\sqrt{3},-\sqrt{3})$. The selection of the sampling points should follow some principles, including that the sampling points should be chosen as close as possible to the origin and it should be tried to make them symmetrical about the origin. For the random field of Gaussian distribution, the collocation point should include the origin, and the number of sampling points is generally twice as the expansion terms of the polynomial chaos. Then, the unknown coefficients are obtained by the collocation approximation method, and the specific formula satisfies 


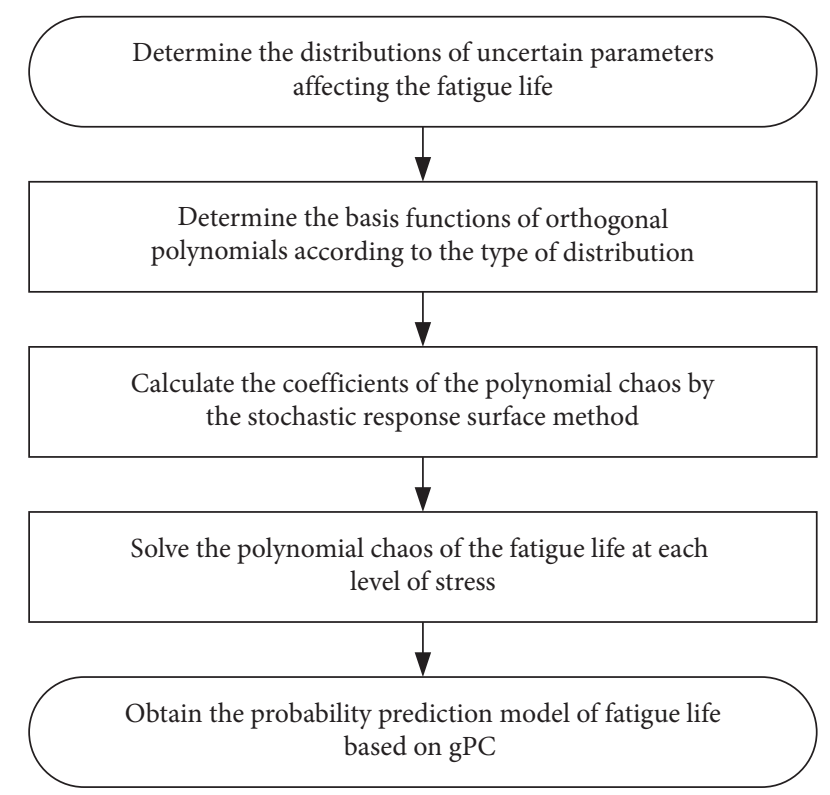

FIgURE 1: The flow chart of probabilistic fatigue life prediction based on gPC.

TABle 4: The Hermite primary functions.

$$
\begin{aligned}
& \Gamma_{0}(x)=1 \\
& \Gamma_{1}(x)=x \\
& \Gamma_{2}(x)=(1 / \sqrt{2})\left(x^{2}-1\right) \\
& \Gamma_{3}(x)=(1 / \sqrt{6})\left(x^{3}-3 x\right) \\
& \Gamma_{4}(x)=(1 / 2 \sqrt{6})\left(x^{4}-6 x^{2}+3\right) \\
& \Gamma_{5}(x)=(1 / 2 \sqrt{30})\left(x^{5}-10 x^{3}+15 x\right) \\
& \Gamma_{6}(x)=(1 / 12 \sqrt{5})\left(x^{6}-15 x^{4}+45 x^{2}-15\right) \\
& \left(\begin{array}{cccc}
\Pi_{0}\left(\eta_{0}\right) & \Pi_{1}\left(\eta_{0}\right) & \ldots & \Pi_{s-1}\left(\eta_{0}\right) \\
\Pi_{0}\left(\eta_{1}\right) & \Pi_{1}\left(\eta_{1}\right) & \ldots & \Pi_{s-1}\left(\eta_{1}\right) \\
\vdots & \vdots & \vdots & \vdots \\
\Pi_{0}\left(\eta_{N}\right) & \Pi_{1}\left(\eta_{N}\right) & \ldots & \Pi_{s-1}\left(\eta_{N}\right)
\end{array}\right)\left(\begin{array}{c}
\beta_{0} \\
\beta_{1} \\
\vdots \\
\beta_{s-1}
\end{array}\right)=\left(\begin{array}{c}
Y\left(\eta_{0}\right) \\
Y\left(\eta_{1}\right) \\
\vdots \\
Y\left(\eta_{N}\right)
\end{array}\right)
\end{aligned}
$$

where $\eta_{0}, \eta_{1}, \ldots, \eta_{N}$ are the sampling points and $N$ is the number of the sampling points.

The whole process of solving the expansion coefficients of polynomials by the random response surface method can be represented by the flow chart shown in Figure 2 .

\section{An Illustrative Example}

To verify the feasibility of the proposed probabilistic fatigue life prediction method, two different nonlinear fatigue $\mathrm{cu}-$ mulative damage models including the improved Manson-Halford model [43] and the improved Corten-Dolan model [44] are used to predict the probabilistic fatigue life, respectively. The prediction results are compared with those of the Monte Carlo method. The probabilistic fatigue life prediction of aluminum alloy welded joints is carried out considering the uncertainty of the S-N curve. Constant amplitude loading or two-stage loading mode is usually an ideal load-bearing state, but in engineering practice, the load

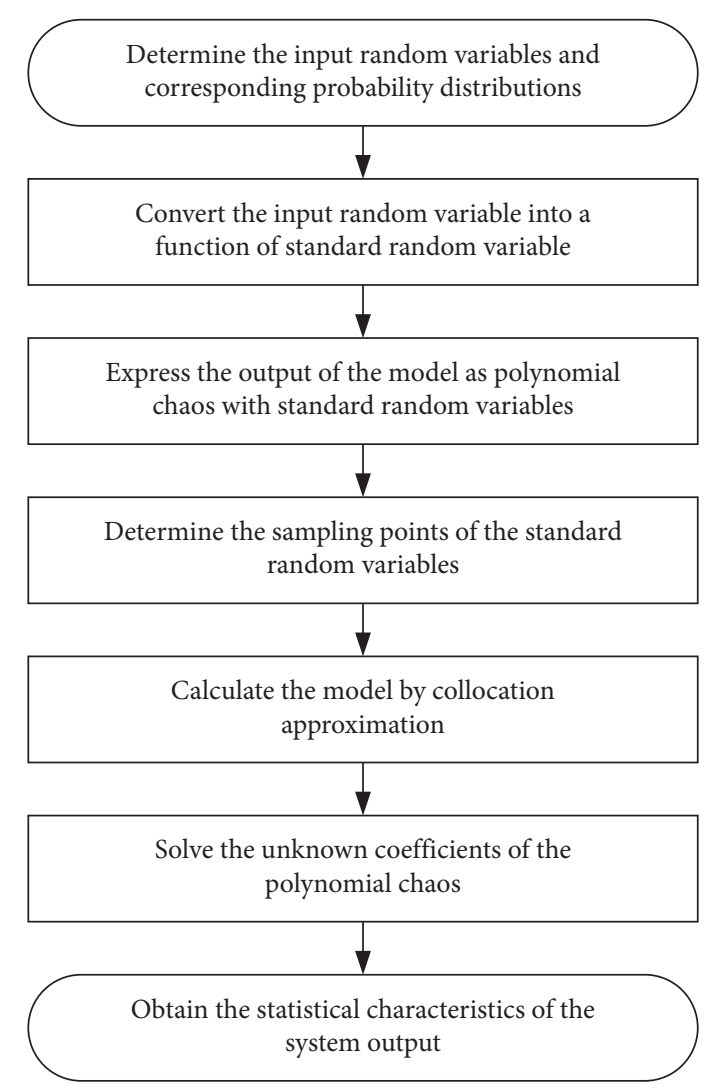

FIgURE 2: The implementation process of the stochastic response surface method.

of welded joints is more complex and changeable. Therefore, this section will predict the probabilistic fatigue life of welded joints under multistage variable amplitude loading on the basis of gPC, the improved Manson-Halford model, and the improved Corten-Dolan model. The data in this section are from the fatigue tests of 45 \# steel welded joints under constant amplitude and program block loads. A total of five groups of 10 specimens were tested under constant amplitude loading, and the test fatigue life under applied stress amplitude and all levels of load is shown in Table 5. The program block load is loaded by multistage variable amplitude load, and the amplitude and cycle times of all levels of stress are shown in Figure 3. A total of 6 specimens are used to carry out the program block load test, and the fatigue life obtained is shown in Table 6.

First of all, the statistical characteristics of the parameters $C$ and $m$ which affect the fatigue life are analyzed according to Table 5 . There are 5 groups of fatigue tests carried out in reference [45]. Taking one from the 10 test data under each stress amplitude, a group of $C$ and $m$ can be obtained by fitting. There are 50 experimental data here, and there are a total of $\left(C_{10}^{1}\right)^{5}=10^{5}$ groups. It is not realistic to combine and fit all the forms. Therefore, 200 combinations are randomly selected for statistical analysis of $C$ and $m$. The statistical results are shown in Figures 4 and 5, where $\ln (C)$ and $m$ follow normal distributions. Therefore, the influence of the uncertainty of the S-N curve on the fatigue life can be analyzed by the Hermite polynomial chaos expansion, as shown in Figures 6 and 7. 
TABLe 5: The fatigue life test data of a 45 \# steel welded joint under constant amplitude load.

\begin{tabular}{|c|c|c|c|c|c|}
\hline \multirow{2}{*}{ Group ID } & \multicolumn{5}{|c|}{ Experiment life $\left(N_{f} / 105\right)$ (105 cycles) } \\
\hline & $\Delta \sigma=750 \mathrm{MPa}$ & $\Delta \sigma=650 \mathrm{MPa}$ & $\Delta \sigma=630 \mathrm{MPa}$ & $\Delta \sigma=590 \mathrm{MPa}$ & $\Delta \sigma=520 \mathrm{MPa}$ \\
\hline 1 & 0.2080 & 0.4940 & 0.7089 & 1.3219 & 1.9670 \\
\hline 2 & 0.2215 & 0.8175 & 0.9041 & 1.4129 & 2.0951 \\
\hline 3 & 0.2430 & 0.8590 & 0.9281 & 1.5269 & 3.2367 \\
\hline 4 & 0.2550 & 0.9629 & 0.9430 & 1.5678 & 4.5625 \\
\hline 5 & 0.2700 & 1.0200 & 0.9616 & 1.6421 & 4.6132 \\
\hline 6 & 0.2900 & 1.0869 & 1.0030 & 1.7002 & 4.7381 \\
\hline 7 & 0.3470 & 1.1269 & 1.1981 & 1.7848 & 5.2336 \\
\hline 8 & 0.3780 & 1.1830 & 1.2020 & 1.9280 & 5.4815 \\
\hline 9 & 0.4610 & 1.1929 & 1.5321 & 2.4638 & 6.5569 \\
\hline 10 & 0.6100 & 1.6850 & 1.8919 & 2.6272 & 12.9211 \\
\hline
\end{tabular}

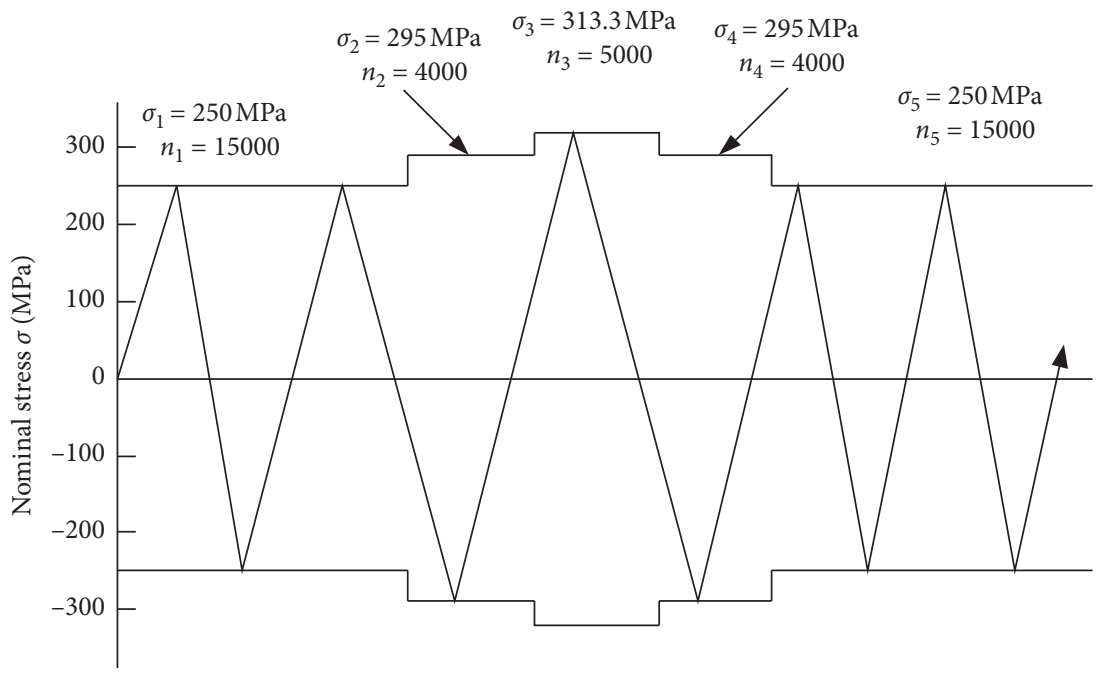

FIgURE 3: The schematic diagram of multistage variable amplitude loading for a 45 \# steel welded joint.

TABLE 6: The fatigue life test data of a 45 \# steel welded butt joint under multistage variable amplitude load.

\begin{tabular}{lcc}
\hline Group ID & Experimental value of fatigue life (load block) & \multicolumn{2}{c}{$\begin{array}{c}\text { Predicted value of fatigue life (load block) } \\
\text { Modified Corten-Dolan model }\end{array}$} \\
\hline 1 & 3.717 & 9.3918 \\
2 & 5.189 & \\
3 & 6.729 & \\
4 & 7.452 & \\
5 & 7.943 & \\
6 & 12.151 & \\
\hline
\end{tabular}

The probability of each predicted value can be judged from Figures 6 and 7, that is, when the uncertainty of the S-N curve is considered, the fatigue life predicted by the improved Manson-Halford model and the improved Corten-Dolan model has a certain uncertainty, but not a definite value. This can provide a more reasonable explanation for the abovementioned phenomenon, that is, the welded joint designed according to the model with high prediction accuracy may still have fatigue failure during its safety period.

The gPC method uses the addition and subtraction algorithm to express the dispersion and randomness of fatigue life caused by the uncertainty of material parameters $C$ and $m$ under constant amplitude load, so as to conside the uncertainty of the S-N curve. Compared with the power function form, the calculation is faster. However, compared with the Monte Carlo method, the polynomial chaos theory has a limitation, that is, the calculation process of the polynomial chaos expansion method is more complex and tedious when there are more parameters, and the calculation efficiency is higher when the parameters are less. Therefore, in the fatigue life prediction of welded joints, if there are more uncertain parameters input, the polynomial chaos theory still needs to be studied more deeply in order to simplify the calculation process and save time and cost. In the analysis of this chapter, the uncertainty of the S-N curve is due to the dispersion and randomness of the two material 


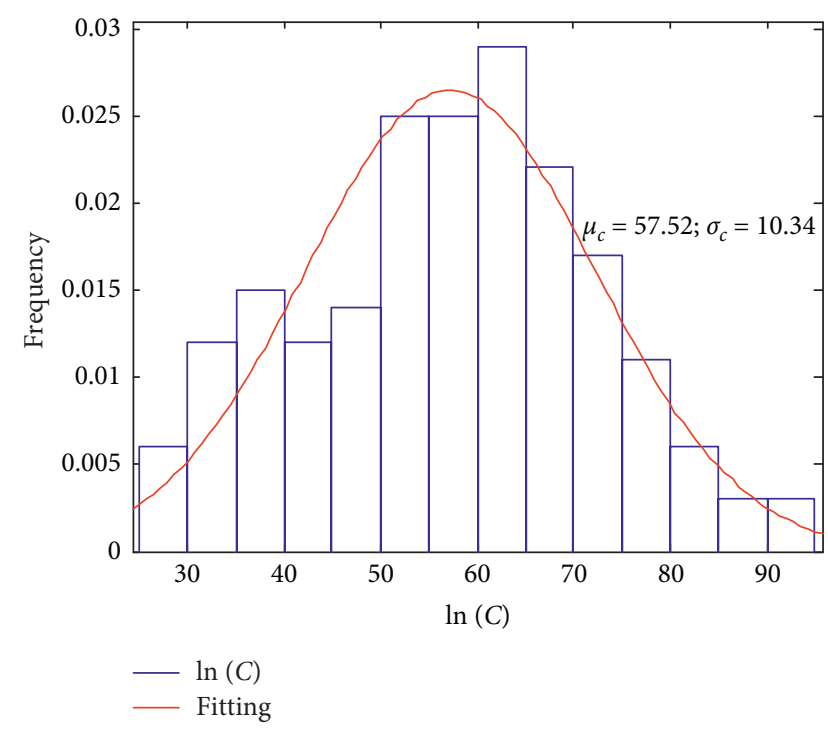

FIgUre 4: The statistical distribution results for $C$ of the S-N curve (45 \# steel welded butt joint).

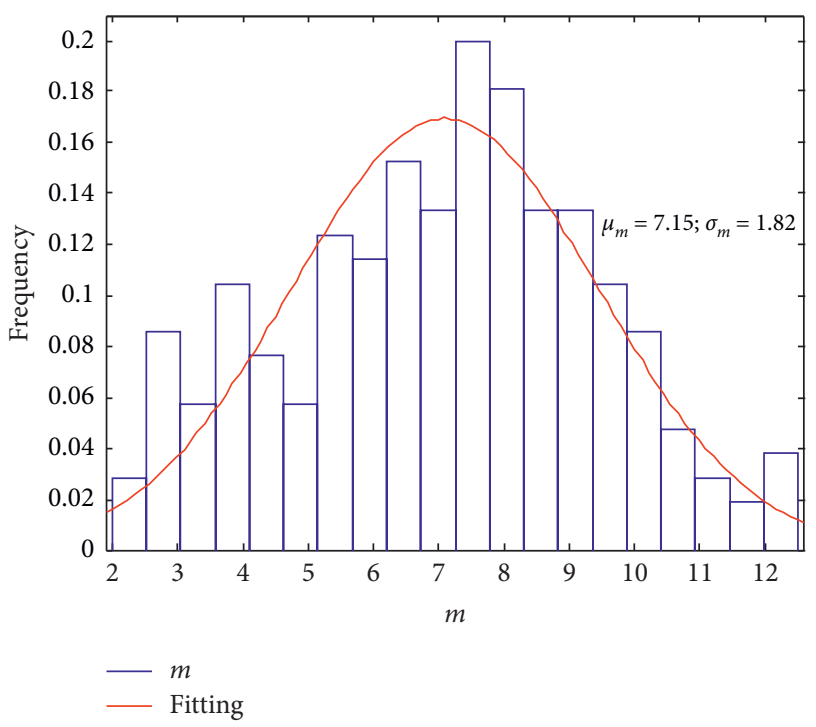

FIgURE 5: The statistical distribution results for $m$ of the S-N curve (45 \# steel welded butt joint).

parameters. In the process of calculation, it is found that the fatigue life prediction under second-order and multistage loading by the gPC method is more efficient than that by the Monte Carlo method, and the prediction results are close. Therefore, the fatigue life prediction method based on gPC expansion and considering the uncertainty of the S-N curve proposed in this chapter can obtain satisfactory probabilistic life prediction results through a simple and efficient calculation process.

By comparing the probabilistic fatigue life of a 45 steel welded butt joint obtained by the gPC expansion method and Monte Carlo simulation method under multistage variable amplitude block load, a conclusion similar to that of second-order loading mode can be obtained, that is, the probabilistic fatigue life prediction result of polynomial

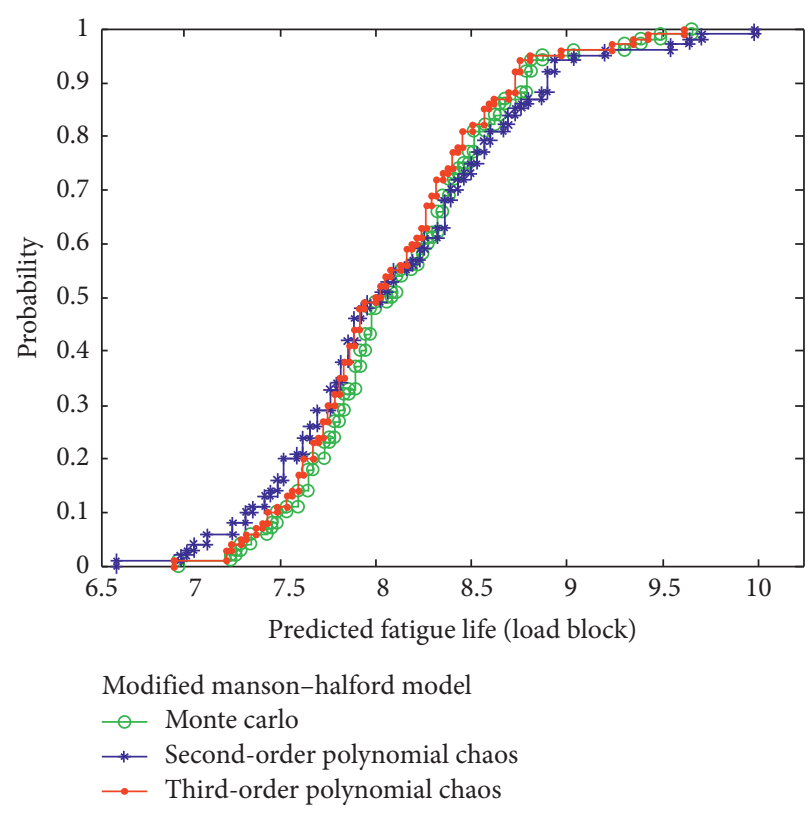

FIGURE 6: The probabilistic fatigue life prediction results under the modified Manson-Halford model (45 \# steel welded butt joints).

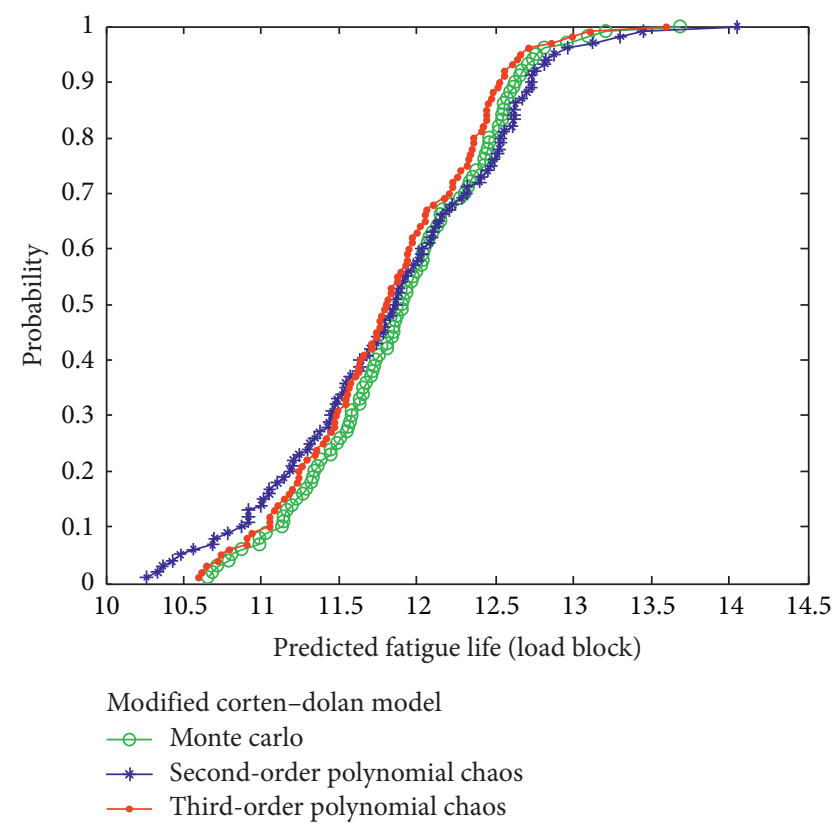

FIgURE 7: The probabilistic fatigue life prediction results under the modified Corten-Dolan model (45 \# steel welded butt joints).

chaos expansion is quite close to that of the Monte Carlo method. This shows that the polynomial chaos theory is applicable in the field of fatigue life prediction and is an effective method to reflect and characterize the uncertainty of the S-N curve.

\section{Summary and Conclusions}

Based on the gPC, the uncertain parameters that affect the fatigue life under constant amplitude load are regarded as 
random variables to consider the uncertainty of the S-N curve of welded joints. Combined with the improved Manson-Halford model and the improved Corten-Dolan model, the probabilistic fatigue life of welded joints is predicted. By comparing the results of probabilistic fatigue life prediction under secondary and multistage load, it is found that the probabilistic fatigue life prediction method based on gPC is close to the Monte Carlo method when it reaches the second or third order. Therefore, this method can be applied to fatigue life prediction under secondary or more complex loading conditions. The polynomial chaotic expansion method expresses the fatigue life as the polynomial of the addition and subtraction rule, and the calculation efficiency is higher than that of the Monte Carlo method, which verifies the feasibility of gPC expansion in fatigue life prediction, broadens the engineering application field of polynomial chaos theory, and provides a more practical way to deal with the uncertainty in the process of life prediction.

\section{Data Availability}

The data used to support the findings of this study are included within the article.

\section{Conflicts of Interest}

The authors declare no conflicts of interest.

\section{Authors' Contributions}

H. G. and X. Z. conceptualized the work; H. G. formulated the methodology, conducted formal analysis and investigation, collected resources, prepared the original draft, reviewed and edited the manuscript, was involved in project administration, and acquired funding; X. Z. was responsible for the software, curated data, and performed visualization; H. G., X. Z., X. Y., and B. Z. performed validation; and X. Y. and B. Z. supervised the work. All authors have read and agreed to the published version of the manuscript.

\section{Acknowledgments}

This research was supported by the Sichuan Science and Technology Program, grant nos. 2019YJ0395 and 2021YJ0519, and General Aviation Special Research Project of Civil Aviation Flight University of China, grant no. THZX2018-09. The funders had no role in the design of the study; in the collection, analyses, or interpretation of data; in the writing of the manuscript; or in the decision to publish the results.

\section{References}

[1] W.-f. Xu and Z.-1. Zhang, "Microstructure and mechanical properties of laser beam welded TC4/TA15 dissimilar joints," Transactions of Nonferrous Metals Society of China, vol. 26, no. 12, pp. 3135-3146, 2016.

[2] B. Jonsson, Z. Barsoum, and J.-O. Sperle, "Weight optimization and fatigue design of a welded bogie beam structure in a construction equipment," Engineering Failure Analysis, vol. 19, pp. 63-76, 2012.
[3] S. A. Oosterhof and R. G. Driver, "Effects of connection geometry on block shear failure of welded lap plate connections," Journal of Constructional Steel Research, vol. 67, no. 3, pp. 525-532, 2011.

[4] J.-C. He, S.-P. Zhu, D. Liao, and X.-P. Niu, "Probabilistic fatigue assessment of notched components under size effect using critical distance theory," Engineering Fracture Mechanics, vol. 235, Article ID 107150, 2020.

[5] Y. Ai, S. P. Zhu, D. Liao et al., "Probabilistic modeling of fatigue life distribution and size effect of components with random defects," International Journal of Fatigue, vol. 126, pp. 165-173, 2019.

[6] X.-P. Niu, R.-Z. Wang, D. Liao, S.-P. Zhu, X.-C. Zhang, and B. Keshtegar, "Probabilistic modeling of uncertainties in fatigue reliability analysis of turbine bladed disks," International Journal of Fatigue, vol. 142, Article ID 105912, 2021.

[7] K. B. Rao, M. Anoop, G. Raghava, M. Prakash, and A. Rajadurai, "Probabilistic fatigue life analysis of welded steel plate railway bridge girders using S-N curve approach," Proceedings of the Institution of Mechanical Engineers, Part O: Journal of Risk and Reliability, vol. 227, no. 4, pp. 385-404, 2013.

[8] H. Shen, J. Lin, and E. Mu, "Probabilistic model on stochastic fatigue damage," International Journal of Fatigue, vol. 22, no. 7, pp. 569-572, 2000.

[9] Y. Liu and S. Mahadevan, "Stochastic fatigue damage modeling under variable amplitude loading," International Journal of Fatigue, vol. 29, no. 6, pp. 1149-1161, 2007.

[10] K. Ni and S. Mahadevan, "Strain-based probabilistic fatigue life prediction of spot-welded joints," International Journal of Fatigue, vol. 26, no. 7, pp. 763-772, 2004.

[11] Y. Liu and S. Mahadevan, "Probabilistic fatigue life prediction using an equivalent initial flaw size distribution," International Journal of Fatigue, vol. 31, no. 3, pp. 476-487, 2009.

[12] J. Maljaars, H. M. G. M. Steenbergen, and A. C. W. M. Vrouwenvelder, "Probabilistic model for fatigue crack growth and fracture of welded joints in civil engineering structures," International Journal of Fatigue, vol. 38, pp. 108-117, 2012.

[13] J. Luo and P. Bowen, "A probabilistic methodology for fatigue life prediction," Acta Materialia, vol. 51, no. 12, pp. 3537-3550, 2003.

[14] G. Yi and M. P. Norton, "Materials fatigue life distribution: a maximum entropy approach," Journal of Testing and Evaluation, vol. 26, no. 1, pp. 53-63, 1998.

[15] X. Guan, R. Jha, and Y. Liu, "Probabilistic fatigue damage prognosis using maximum entropy approach," Journal of Intelligent Manufacturing, vol. 23, no. 2, pp. 163-171, 2012.

[16] X. Guan, Y. Liu, R. Jha, A. Saxena, J. Celaya, and K. Geobel, "Comparison of two probabilistic fatigue damage assessment approaches using prognostic performance metrics," International Journal of Prognostics and Health Management, vol. 5, no. 1, 2011.

[17] S.-P. Zhu, H.-Z. Huang, V. Ontiveros, L.-P. He, and M. Modarres, "Probabilistic low cycle fatigue life prediction using an energy-based damage parameter and accounting for model uncertainty," International Journal of Damage Mechanics, vol. 21, no. 8, pp. 1128-1153, 2012.

[18] M. Naderi and M. Khonsari, "Real-time fatigue life monitoring based on thermodynamic entropy," Structural Health Monitoring, vol. 10, no. 2, pp. 189-197, 2011.

[19] T. Temfack and C. Basaran, "Experimental verification of thermodynamic fatigue life prediction model using entropy as 
damage metric," Materials Science and Technology, vol. 31, no. 13, pp. 1627-1632, 2015.

[20] J. Wang and Y. Yao, "An entropy-based failure prediction model for the creep and fatigue of metallic materials," Entropy, vol. 21, no. 11, p. 1104, 2019.

[21] J. Kim, J. Yi, J. Kim, G. Zi, and J. S. Kong, "Fatigue life prediction methodology using entropy index of stress interaction and crack severity index of effective stress," International Journal of Damage Mechanics, vol. 22, no. 3, pp. 375-392, 2013.

[22] X. Guan, R. Jha, and Y. Liu, "Model selection, updating, and averaging for probabilistic fatigue damage prognosis," Structural Safety, vol. 33, no. 3, pp. 242-249, 2011.

[23] D. An, J.-H. Choi, N. H. Kim, and S. Pattabhiraman, "Fatigue life prediction based on Bayesian approach to incorporate field data into probability model," Structural Engineering and Mechanics, vol. 37, no. 4, pp. 427-442, 2011.

[24] M. Chookah, M. Nuhi, and M. Modarres, "A probabilistic physics-of-failure model for prognostic health management of structures subject to pitting and corrosion-fatigue," Reliability Engineering \& System Safety, vol. 96, no. 12, pp. 1601-1610, 2011.

[25] B. A. Zárate, J. M. Caicedo, J. Yu, and P. Ziehl, "Bayesian model updating and prognosis of fatigue crack growth," Engineering Structures, vol. 45, pp. 53-61, 2012.

[26] H.-Z. Huang, M. J. Zuo, and Z.-Q. Sun, "Bayesian reliability analysis for fuzzy lifetime data," Fuzzy Sets and Systems, vol. 157 , no. 12 , pp. 1674-1686, 2006.

[27] G. Mustafa, C. Crawford, and A. Suleman, "Fatigue life prediction of laminated composites using a multi-scale M-LaF and Bayesian inference," Composite Structures, vol. 151, pp. 149-161, 2016.

[28] S.-P. Zhu, H.-Z. Huang, R. Smith, V. Ontiveros, L.-P. He, and M. Modarres, "Bayesian framework for probabilistic low cycle fatigue life prediction and uncertainty modeling of aircraft turbine disk alloys," Probabilistic Engineering Mechanics, vol. 34, pp. 114-122, 2013.

[29] A. Muc, "Fuzzy approach in modeling static and fatigue strength of composite materials and structures," Neurocomputing, vol. 393, pp. 156-164, 2020.

[30] Z. M. Nopiah, M. H. Osman, S. Abdullah, and M. N. Baharin, "The identification of low fatigue damage using Fuzzy double clustering framework," in Proceedings of the 2011 IEEE 7th International Colloquium on Signal Processing and its Applications (CSPA), pp. 181-186, Penang, Malaysia, March 2011.

[31] S. Bhalla, P. A. Vittal, and M. Veljkovic, "Piezo-impedance transducers for residual fatigue life assessment of bolted steel joints," Structural Health Monitoring: An International Journal, vol. 11, no. 6, pp. 733-750, 2012.

[32] S.-P. Zhu, H.-Z. Huang, and Z.-L. Wang, "Fatigue life estimation considering damaging and strengthening of low amplitude loads under different load sequences using fuzzy sets approach," International Journal of Damage Mechanics, vol. 20, no. 6, pp. 876-899, 2011.

[33] S.-P. Zhu, H.-Z. Huang, W. Peng, H.-K. Wang, and S. Mahadevan, "Probabilistic physics of failure-based framework for fatigue life prediction of aircraft gas turbine discs under uncertainty," Reliability Engineering \& System Safety, vol. 146, pp. 1-12, 2016.

[34] Y. Ren, D. Gao, and L. Xu, "Prediction of service life of large centrifugal compressor remanufactured impeller based on clustering rough set and fuzzy Bandelet neural network," Applied Soft Computing, vol. 78, pp. 132-140, 2019.
[35] D. Zhao, C. Gao, Z. Zhou, S. Liu, B. Chen, and J. Gao, "Fatigue life prediction of the wire rope based on grey theory under small sample condition," Engineering Failure Analysis, vol. 107, Article ID 104237, 2020.

[36] Y. Xu, L. Mili, A. Sandu, M. R. von Spakovsky, and J. Zhao, "Propagating uncertainty in power system dynamic simulations using polynomial chaos," IEEE Transactions on Power Systems, vol. 34, no. 1, pp. 338-348, 2018.

[37] S. Yin, D. Yu, H. Yin, and B. Xia, "A new evidence-theorybased method for response analysis of acoustic system with epistemic uncertainty by using Jacobi expansion," Computer Methods in Applied Mechanics and Engineering, vol. 322, pp. 419-440, 2017.

[38] P. K. Singh, "Complex neutrosophic concept lattice and its applications to air quality analysis," Chaos, Solitons \& Fractals, vol. 109, pp. 206-213, 2018.

[39] N. Wiener, "The homogeneous chaos," American Journal of Mathematics, vol. 60, no. 4, pp. 897-936, 1938.

[40] R. Ghanem, "Stochastic finite elements with multiple random non-Gaussian properties," Journal of Engineering Mechanics, vol. 125, no. 1, pp. 26-40, 1999.

[41] D. Xiu, Generalized (Wiener-Askey) Polynomial Chaos, Brown University, Providence, RI, USA, 2004.

[42] K. Sepahvand, S. Marburg, and H.-J. Hardtke, "Uncertainty quantification in stochastic systems using polynomial chaos expansion," International Journal of Applied Mechanics, vol. 2, no. 2, pp. 305-353, 2010.

[43] H. Gao, H. Z. Huang, S. P. Zhu, Y. F. Li, and R. Yuan, “A modified nonlinear damage accumulation model for fatigue life prediction considering load interaction effects," The Scientific World Journal, vol. 2014, Article ID 164378, 2014.

[44] H. Gao, H.-Z. Huang, Z. Lv, F.-J. Zuo, and H.-K. Wang, “An improved Corten-Dolan's model based on damage and stress state effects," Journal of Mechanical Science and Technology, vol. 29, no. 8, pp. 3215-3223, 2015.

[45] J. Yan, X. L. Zheng, and K. Zhao, "Prediction of fatigue life and its probability distribution of notched friction welded joints under variable-amplitude loading," International Journal of Fatigue, vol. 22, no. 6, pp. 481-494, 2000. 